\title{
What is the OPTiMal Way to Manage In-Transit Disease?
}

\author{
Kelly Olino, MD and Douglas S. Tyler, MD \\ Department of Surgery, The University of Texas Medical Branch, Galveston, TX
}

\section{CONCEPT}

Intratumoral treatment as a form of regional immunotherapy can trace its origin back to 1892 with the description of Coley's toxin. ${ }^{1}$ Historically, given the absence of effective systemic therapies for melanoma, regional therapeutics have played a prominent role targeting regionally advanced disease, most commonly in-transit disease. In-transit disease, the multifocal occurrence of tumor nodules in various layers of the skin, arises between the primary tumor and regional nodal basin. Despite its aggressive appearance, in many cases in-transit disease remains regionally confined for prolonged periods of time, making regional therapeutics, ranging from intralesional Bacillus Calmette-Guerin (BCG) to isolated limb perfusion/infusion, destination therapies. Over the last 5 years, as systemic treatments, including targeted therapies and checkpoint blockade inhibitors, have demonstrated significant effectiveness on metastatic disease, some have called into question the role of regional therapeutics in managing patients with regionally advanced stage IIIb and IIIc disease. The recent approval of talimogene laherparepvec (TVEC) following the OPTiM III trial, ${ }^{2}$ a phase III, randomized, prospective drug registration trial, further complicates the debate by opening a new chapter of oncolytic immunotherapy in regional therapeutics.

T-VEC is an attenuated herpes simplex virus (HSV-1) designed to be directly injected into, and selectively replicate in, cancer cells while secreting granulocytemacrophage colony-stimulating factor (GM-CSF). In the OPTiM III trial, T-VEC met its primary endpoint, showing a statistically significant durable response rate of (16\%) when injected into tumor nodules compared with GM-CSF

(C) Society of Surgical Oncology 2016

First Received: 10 May 2016;

Published Online: 5 July 2016

D. S. Tyler, MD

e-mail: dstyler@utmb.edu alone injected subcutaneously (2\%) in patients with unresectable stage IIIb, IIIc, or IV melanoma; however, the trial did not demonstrate a statistically significant difference in overall survival between the two arms.

T-VEC primarily works by direct cytolytic effects of the herpes virus in virally-infected tumor cells coupled by downstream effects that generate a significant local immune response that is elicited following cell death when viral and tumor-specific epitopes are released in the presence of GM-CSF. The viral antigens serve as danger signals, known as damage-associated molecular pattern molecules (DAMPs) or pathogen-associated molecular pattern molecules (PAMPs) which initiate an immune inflammatory response, while GM-CSF is thought to recruit and mature dendritic cells to the area of inflammation. In theory, this creates an immune environment culminating in $\mathrm{T}$ cell-mediated tumor-specific killing capable of generating responses both locally and at distant metastatic sites. ${ }^{3}$ As such, regional treatments may now be able to be designed to kill tumors in a way to generate a maximal systemic impact.

\section{TRIAL DESIGN}

Although we applaud the authors for such a tremendous undertaking and taking a novel platform from the bench to human clinical trials, there are several concerns we have about the trial design and interpretation of data that will need further clarification before we can clearly say where this treatment modality should be utilized in the context of various therapeutic options currently available to patients with stage IIIb, IIIc, and IVa disease.

Trials such as the OPTiM III are challenging to design and carry out given the complex nature of in-transit disease with its multiple lesions and the need for serial measurements. Frequently, in-transit lesions are less than $1 \mathrm{~cm}$ and are not visible on imaging studies. The close proximity of lesions to each other can make measurements even with calipers and photodocumentation difficult. Furthermore, a single lesion can be made up of several smaller lesions that 
grow together. Defining response as a large lesion of this nature regresses into several smaller ones can be challenging.

The OPTiM III study inclusion criterion required measureable disease with at least a solitary, injectable lesion $\geq 1 \mathrm{~cm}$ or an aggregate of lesions $\geq 1 \mathrm{~cm}$ in either the nodal, subcutaneous, or dermal space. Treatment then began with the largest accessible tumor treated, followed by smaller injectable lesions. When choosing an intralesional treatment, we feel it is important to know the response by lesion size as well as by the location of the tumor in order to allow for appropriate patient selection. Although the authors did not stratify treatment responses of injected lesions by size, the US FDA, during their review of the T-VEC platform in their briefing document, stratified patients who experienced a durable response by size of the largest lesion. ${ }^{4}$ It appears that patients who had smaller tumors likely derived the greatest benefit. There were 29 patients with tumors of greatest diameter $<1 \mathrm{~cm}, 14$ of whom (48\%) achieved durable responses. Of the 34 patients with the largest tumor measuring $1-2 \mathrm{~cm}, 11$ (32\%) had a durable response, while of the 226 patients with a tumorn $>2 \mathrm{~cm}$, only 21 were durable responders (9.3\%).

The authors performed subset analysis and reported the patterns of response for (i) directly treated lesions, (ii) noninjected, non-visceral lesions, and (iii) non-injected visceral lesions, and conclude that off-site target responses are immune-mediated. However, the authors did not stratify treatment responses of individual injected lesions by their location in the nodal, subcutaneous, or dermal spaces. Recent research has pointed to differences in the resident immune populations within the skin and how this impacts the relative immunogenicity of tumors. The subcutaneous space is rich in macrophages and monocytes, whereas the dermis is rich in multiple populations of dendritic cells, which are key for the generation of any proposed cellmediated immune response. ${ }^{5,6}$ Given that animal studies suggest immunogenic response may be different to ablative therapies based on tumor location, with dermally based tumors generating more robust immune responses than subcutaneously placed tumors, future regional therapy trials may need to focus more on the location of regionally treated tumor within the architecture of the skin.

For these same reasons, the selection of subcutaneously administered GM-CSF as the control arm, which was previously only found to have efficacy in the adjuvant setting, seems curious compared with using other direct intralesional treatments that are known to have some regional efficacy. Furthermore, although the study design of the T-VEC arm of the trial called for treatment to be administered for 24 weeks and to allow for 'pseudo-progression' of disease given known delayed treatment responses with immunotherapy treatments, this window does not seem to have been afforded for the GM-CSF arm alone. The median duration of T-VEC was 23 weeks and only 10 weeks for GM-CSF. ${ }^{2}$ There were also many more early withdrawals among the GM-CSF arm (56\% at 3 months and $75 \%$ at 6 months) compared with the TVEC arm (29\% at 3 months and $58 \%$ at 6 months). ${ }^{4}$ Use of another intralesional therapy in the control arm, instead of GM-CSF, would have been a better way to examine what incremental benefit, if any, T-VEC had in this subgroup of patients who progressed with new lesions.

The investigators also reported responses in non-injected, non-visceral lesions, with $34 \%$ (331/981) of lesions having a response $>50 \%$. Forty-eight percent were in the same body site and $29 \%$ were not assigned a body site. Only $23 \%$ (77/331) were located in a 'different body site', but this was not defined. In the description of the initial study design, the body was divided up into 42 separate areas on a grid. From how the data are presented in the manuscript, it is not clear if a different body site represents areas separated by a few centimeters or lesions that are truly further away with no shared dermal lymphatics. This would be helpful in trying to tease out what may truly be an immune-mediated phenomena versus direct virus spread via blood or lymphatics to neighboring lesions.

One of the more intriguing findings in this study was that $15 \%(27 / 177)$ of distant visceral lesions, mainly in the lung, had $>50 \%$ treatment response. This provides some support for an immune-generated mechanism of action. Further studies showing the immune responses in these patients are eagerly anticipated to build on previous work in the phase II studies that showed increased responses in MART-1-specific CD8 T cells. ${ }^{7}$

If the effects of T-VEC are immune-mediated secondary to immunogenic tumor cell death and GM-CSF being released in the same microenvironment, the biggest potential of this new compound lies in combination with immunotherapy treatment strategies. This premise is supported by preliminary data from a phase $1 \mathrm{~b} / 2$ clinical trial presented at ASCO 2015, ${ }^{8}$ which showed that T-VEC combined with ipilimumab had an overall response rate of $56 \%$ (33\% complete response) and a durable response rate of $44 \%$. Studies such as these, using combination therapies will continue to advance the field and head-tohead comparisons of immune coreceptor blockade in combination with regional chemotherapy or other intralesional treatments such as PV-10 (Rose bengal) should continue to be pursued. Several examples of these combination strategies already exist, including National Cancer Institute (NCI) trial 0262600 looking at T-VEC and pembrolizumab, NCI trial 01323517 combining isolated limb infusion using melphalan with ipilimumab, and NCI trial 02115243, which is being modified to look at combining 
nivolumab and ipilimumab neoadjuvantly prior to isolated limb infusion with melphalan.

\section{PATIENT SELECTION}

The issue of appropriate patient selection also leads to some practical concerns about the T-VEC trial. There is difficulty applying the treatment methodology of the investigators into clinical practice based on descriptions of what constituted 'unresectable' disease and the heterogeneous manner in which treatment was administered. Furthermore, treatment was administered in such a way to favor treating new lesions first and then established lesions, even if the established lesions were larger. We need to be cautious when interpreting the data since, inadvertently, such an approach could lead changing the overall reported treatment responses (i.e. easier to get a $>50 \%$ response for a $6 \mathrm{~mm}$ tumor than for a $2 \mathrm{~cm}$ tumor).

Despite the favorable safety profile published in the OPTiM trial compared with systemic immune checkpoint blockade therapy, there are no published data on viral shedding and potential for transmission to healthcare workers, close contacts, immunocompromised or pregnant bystanders. While others have demonstrated that oncolytic herpes viruses can be used safely in humans, ${ }^{9}$ there is currently a phase II clinical trial underway with T-VEC to address concerns over viral shedding but no results have been published thus far. Storage and administration of TVEC may also be a hurdle at some centers given the need to store at $-70{ }^{\circ} \mathrm{C}$. Finally, the cost for a course of treatment that provides locoregional disease control but not a significant overall survival benefit is projected to be approximately $\$ 65,000 .{ }^{10}$ In contrast, checkpoint blockade therapy, which is associated with improved survival, has a cost ranging between $\$ 100,000$ and $\$ 150,000$. The National Health Service in the UK has deemed T-VEC treatment to not be cost effective ${ }^{11}$ and this may prove to be a significant hurdle in this country as healthcare moves towards a population health management strategy for cancer care.

So where does that leave us for managing patients with in-transit disease? There are multiple approaches to managing patients with stage IIIb, IIIc, and IVa disease, ranging from systemic treatments with checkpoint blockade inhibitors to targeted treatments for individuals who have a BRAF mutation. Regional treatments include surgical excision, intralesional therapy with compounds such as TVEC, and regional chemotherapy approaches if disease is confined to the extremity. There can be significant differences of opinion, even among surgeons, as to the optimal initial approach, as questions as simple as 'How do you define resectable in-transit disease?' vary from practice to practice. There is also frequently a split between medical oncologists and surgical oncologists as to which approach is best. Medical oncologists tend to favor systemic approaches with immunotherapy as a way to try to achieve a long-term complete response. Surgical oncologists, who have historically managed these patients with regional approaches, see excellent long-term disease control in 30$40 \%$ of patients and, as such, frequently desire to delay systemic therapy with its attendant morbidity for when patients actually need it. Our personal approach, is that we feel all patients with stage IIIb, IIIc, and IVa disease should be presented at a multidisciplinary tumor board where local expertise and comfort with different treatment options can be balanced with the unique characteristics of a patient's presentation to tailor a treatment plan for that individual. This treatment plan would not only consider participation in various clinical trials but also consider sequential interventions in the event of initial treatment failure.

\section{CONFLICTS OF INTEREST}

Despite efforts to maintain clinical equipoise during the conduct of a clinical trial, there is always room for potential conflict of interest in a high-stakes corporatesponsored drug registration trial. The role of industry in 'marketing' trials to increase visibility of a therapeutic agent is well-documented. ${ }^{12,13}$ Several studies have demonstrated that not only do industry-sponsored trials have more manuscripts per trial in the literature than nonindustry-sponsored trials, but there are also frequently more subset analyses published from industry-sponsored trial datasets. ${ }^{14}$ As such, similar to ASCO's new policy, ${ }^{15}$ we feel that lead investigators and lead authors should not receive honoraria or consulting fees from companies sponsoring trials in which their compound is being evaluated as a prerequisite for publication in this journal.

\section{SUMMARY}

Overall, T-VEC serves as an important advancement of oncolytic immunotherapy and highlights the progress that has been achieved by researchers in designing therapeutics over the past few decades. While researchers are expanding the armamentarium of agents to use in a novel fashion, we must continue to work towards determining what role, if any, regional treatments play in the rapidly changing landscape of melanoma therapies. This challenge needs to push surgeons leading clinical trials to objectively answer two questions: (i) Can a regional therapeutic generate a clinically effective systemic immune response? (ii) Can regional therapeutics augment the clinical effectiveness of systemically administered immunotherapy? 


\section{REFERENCES}

1. Nauts HC, Swift WE, Coley BL. The treatment of malignant tumors by bacterial toxins as developed by the late William B. Coley, M.D., reviewed in the light of modern research. Cancer Res. 1946;6:205-16.

2. Andtbacka RHI, Kaufman HL, Collichio F, et al. Talimogene laherparepvec improves durable response rate in patients with advanced melanoma. J Clin Oncol. 2015;33(25):2780-8.

3. Kohlhapp FJ, Kaufman HL. Molecular pathways: mechanism of action for talimogene laherparepvec, a new oncolytic virus immunotherapy. Clin Cancer Res. 2016;22(5):1048-54.

4. FDA briefing document. Cellular, Tissue, and Gene Therapies Advisory Committee and Oncologic Drugs Advisory Committee Meeting. 2015.

5. Waithman J, Gebhardt T, Bedoui S. Skin tumor immunity: site does matter for antigen presentation by DCs. Eur J Immunol. 2016;46(3):543-6.

6. Joncker NT, Bettini S, Boulet D, et al. The site of tumor development determines immunogenicity via temporal mobilization of antigen-laden dendritic cells in draining lymph nodes. Eur $\mathbf{J}$ Immunol. 2016;46(3):609-18.

7. Kaufman HL, Kim DW, DeRaffele G, et al. Local and distant immunity induced by intralesional vaccination with an oncolytic herpes virus encoding GM-CSF in patients with stage IIIc and IV melanoma. Ann Surg Oncol. 2010;17(3):718-30.

8. Puzanov I, Andtbacka RH, Minor DR, et al. Survival, safety, and response patterns in a phase $1 \mathrm{~b}$ multicenter trial of talimogene laherparepvec (T-VEC) and ipilimumab (ipi) in previously untreated, unresected stage IIIB-IV melanoma [abstract]. 2015 ASCO Annual Meeting, meeting library. J Clin Oncol. 2015;33.
Available at: http://meetinglibrary.asco.org/content/151884-156. Accessed 4 Apr 2016.

9. Geevarghese SK, Geller DA, de Haan HA, et al. Phase I/II study of oncolytic herpes simplex virus NV1020 in patients with extensively pretreated refractory colorectal cancer metastatic to the liver. Hum Gene Ther. 2010;21(9):1119-28.

10. FDA approves IMLYGIC talimogene laherparepvec as first oncolytic viral therapy in the US [press release]. Available at: http:// www.amgen.com/media/news-releases/2015/10/fda-approvesimlygic-talimogene-laherparepvec-as-first-oncolytic-viral-therapyin-the-us/. Accessed 9 May 2016.

11. NICE consults on "no" recommendation for skin cancer drug talimogene laherparepvec [press release]. Available at: https:// www.nice.org.uk/news/press-and-media/nice-consults-on-\%E2\% $80 \% 98$ no\% $2 \% 80 \% 99$-recommendation-for-skin-cancer-drugtalimogene-laherparepvec. Accessed 9 May 2016.

12. Jairam V, Yu JB. Examination of industry payments to radiation oncologists in 2014 using the Centers for Medicare and Medicaid Services open payments database. Int J Radiat OncolBiol Phys. 2016;94(1):19-26.

13. Ramchandren R, Schiffer CA, Ramchandren R, et al. Recap: pattern of duplicate presentations at national hematology-oncology meetings: influence of the pharmaceutical industry. J Oncol Pract. 2016;12(3):252-3.

14. Tibau A, Bedard PL, Srikanthan A, et al. Author financial conflicts of interest, industry funding, and clinical practice guidelines for anticancer drugs. J Clin Oncol. 2015;33(1):100-6.

15. American Society of Clinical Oncology. American Society of Clinical Oncology policy for relationships with companies: background and rationale. J Clin Oncol. 2013;31(16):2037-42. 